\title{
Medical Image Segmentation Using Artificial Neural Networks
}

\author{
Mostafa Jabarouti Moghaddam ${ }^{1}$ and Hamid Soltanian-Zadeh ${ }^{1,2,3}$ \\ ${ }^{1}$ Control and Intelligent Processing Center of Excellence, Department of Electrical and \\ Computer Engineering, University of Tehran, Tehran, \\ ${ }^{2}$ School of Cognitive Sciences, Institute for Research in Fundamental \\ Sciences (IPM), Tehran, \\ ${ }^{3}$ Image Analysis Lab., Radiology Department, Henry Ford Health System, Detroit, \\ 1,2Iran \\ ${ }^{3}$ Michigan, USA
}

\section{Introduction}

Segmentation of tissues and structures from medical images is the first step in many image analysis applications developed for medical diagnosis. Development of treatment plans and evaluation of disease progression are other applications. These applications stem from the fact that diseases affect specific tissues or structures, lead to loss, atrophy (volume loss), and abnormalities. Consequently, an accurate, reliable, and automatic segmentation of these tissues and structures can improve diagnosis and treatment of diseases. Manual segmentation, although prone to rater drift and bias, is usually accurate but is impractical for large datasets because it is tedious and time consuming. Automatic segmentation methods can be useful for clinical applications if they have: 1) ability to segment like an expert; 2) excellent performance for diverse datasets; and 3) reasonable processing speed.

Artificial Neural Networks (ANNs) have been developed for a wide range of applications such as function approximation, feature extraction, optimization, and classification. In particular, they have been developed for image enhancement, segmentation, registration, feature extraction, and object recognition. Among these, image segmentation is more important as it is a critical step for high-level processing such as object recognition. Multi-Layer Perceptron (MLP), Radial Basis Function (RBF), Hopfield, Cellular, and Pulse-Coupled neural networks have been used for image segmentation. These networks can be categorized into feed-forward (associative) and feedback (auto-associative) networks. MLP, Self-Organized Map (SOM), and RBF neural networks belong to the feed-forward networks while Hopfield, Cellular, and Pulse-Coupled neural networks belong to the feedback networks.

This chapter is organized as follows. Section 2 explains methods that benefit from feedback networks such as Hopfield, Cellular, and Pulse-Coupled neural networks for image segmentation. In Section 3, we review the methods that use feedforward networks such as MLP and RBF neural networks. Then, we present our recent method. In this method, deep brain structures are segmented using Geometric Moment Invariants (GMIs) and MLP neural networks. 


\section{Medical image segmentation using feedback Neural Networks}

Feedback or recurrent networks include feedback loops. These networks are very powerful and can get extremely complicated. Hopfield, Cellular, and Pulse-Coupled neural networks described in this section belong to this category of networks.

\subsection{Hopfield Neural Network}

Hopfield Neural Network (HNN), introduced by Hopfield, (1982), consists of a pool of neurons with connections between each unit. Every neuron connects to other neurons with a weight in the network. All neurons are both input and output neurons. The output is a binary value $(0,1$ or $-1,1)$. In the original form of $H N N$, the state of each neuron is determined by (Hopfield, 1982),

$$
\begin{gathered}
V_{i} \rightarrow 1 \quad \text { if } \quad \sum_{i \neq j} T_{i j} V_{j}>U_{i} \\
V_{i} \rightarrow 0 \quad \text { if } \quad \sum_{i \neq j} T_{i j} V_{j}<U_{i}
\end{gathered}
$$

where $V_{i}$ is the output of neuron $i$ and $U_{i}$ is a threshold value. $T_{i j}$ is the strength of the connection between neurons $i$ and $j$. HNN has a scalar value associated with the given state of the network, called energy, E, of the network. It is defined as

$$
E=-\frac{1}{2} \sum \sum_{i \neq j} T_{i j} V_{i} V_{j}
$$

The constraint $T_{i j}=T_{j i}$ (i.e., symmetric weights) guarantees that the energy function decreases monotonically (Hopfield, 1982; Amartur et al., 1992). In this condition, initial values of neurons lead the network to converge at a local minimum. In some version of HNN, a bias for each neuron is considered and the network energy is determined by

$$
E=-\frac{1}{2} \sum \sum_{i \neq j} T_{i j} V_{i} V_{j}-\sum_{k} I_{k} V_{k}
$$

where $I_{k}$ is a bias term.

The energy function minimization is obtained by solving a set of motion equations (Amartur et al., 1992)

$$
\frac{\partial U_{i}}{\partial t}=-\frac{\partial E}{\partial V_{i}}
$$

where $U_{i}$ is the input of the $i$-th neuron. If the input-output function decreases monotonically and the motion equations are satisfied, the energy function decreases as time passes and converges at a local minimum (Amartur et al., 1992). The energy function is nonconvex and has more than one local minimum.

A primary application of HNN is an associative memory (Wu and Pados, 2000; GiménezMartínez, 2000). The network is able to save a given pattern by choosing a proper set of 
weights. From image segmentation point of view, $\mathrm{HNN}$ consists of $N \times M$ neurons with the pixels as the rows and the classes as the columns. HNN is used as a map between the image pixels and their labels (Amartur et al., 1992) (i.e., assigning $\mathrm{N}$ pixels to $\mathrm{M}$ classes). The assignment of the pixels minimizes the energy function. The criterion function or weights can be based on a metric measure between a pixel and a class. In (Amartur et al., 1992), generalized distance measure between the $k$-th pixel and the centroid of class $\ell$ is determined by

$$
R_{k l}=\left\|X_{k}-\bar{X}_{l}\right\|_{A_{i}^{-1}}
$$

where $X_{k}$ is the P-dimensional feature vector of the $k$-th pixel, $A_{i}$ is a positive definite weighting marix, and $\bar{X}_{l}$ is the P-dimensional centroid for class $l$. The objective function to minimize is given by

$$
E=-\frac{1}{2} \sum_{k=1}^{N} \sum_{l=1}^{N} R_{k l} V_{l k}^{2}
$$

where $R_{k l}$ is a symmetric distance measure. By subtituion (7) into (5), the neuron dynamics can be described by

$$
\frac{d U_{k l}}{d t}=-R_{k l} V_{k l}
$$

In (Amartur et al., 1992), the winner-takes-all neuron is used to construct the input-output function for the $k$-th pixel.

$$
V_{k n}(t+1)= \begin{cases}1 & \text { if } U_{k n}=\operatorname{MAX}\left(U_{k l} ; \forall l\right) \\ 0 & \text { otherwise }\end{cases}
$$

The algorithm for clustering an image is as follows:

1. Initialize the inputs of the neurons randomly.

2. Using (9), calculate the output of the neurons. Assign pixels to classes.

3. Compute the centroid and the covariance matrix for class $l$ as described in (Amartur et al., 1992).

4. Solve equation (8) using Euler's approximation.

5. If there is a significant change in the input of each neuron, repeat from step 2), else, terminate.

If the number of clusters is large, the network may over-classify the image into many disjoint regions. In this condition, a similarity or dissimilarity measure should be used to merge them. Fig. 1 shows an example of clustering a magnetic resonance image (MRI) of the head (Amartur et al., 1992). This network is easily implemented in hardware and its high speed makes it appropriate for real-time applications.

The maximum operator generates a crisp classification. To generate a fuzzy classification, (Lin et al., 1996a; Lin et al. 1996b; Lin et al. 1996c) integrated a fuzzy c-means strategy with the HNN and introduced a Fuzzy Hopfield Neural Network (FHNN). They modified the energy function to include the fuzzy parameters. In this network, all of the neurons on the same row compete and eventually the neuron with the maximum membership to a given 


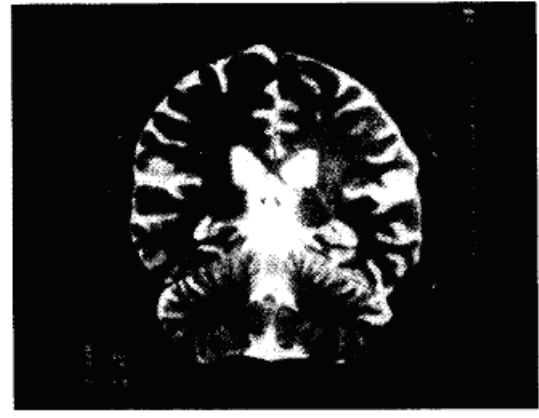

(a)

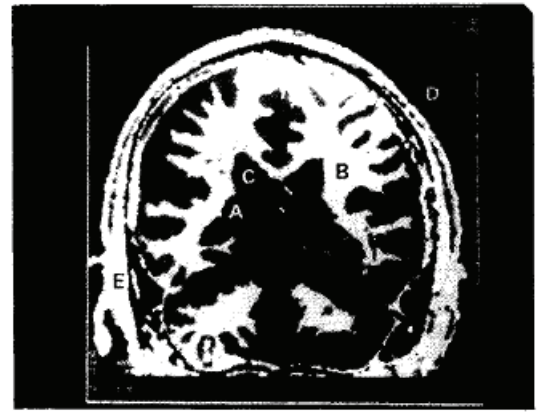

(c)

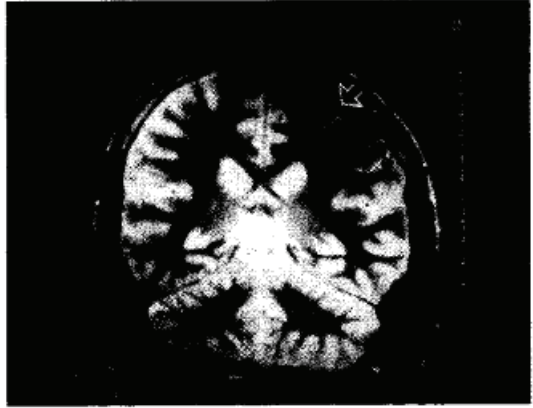

(b)

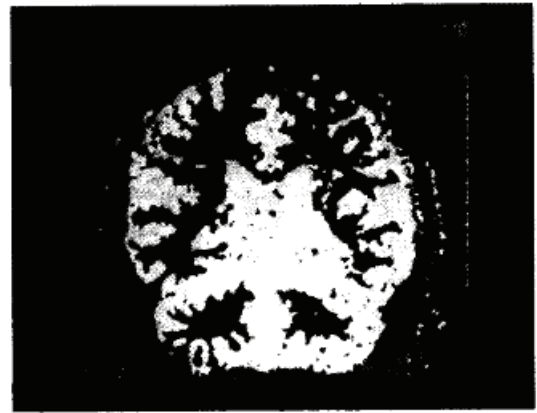

(d)

Fig. 1. Magnetic resonance images of the head of a normal subject: (a) T2-weighted at level 1; (b) proton density-weighted at level 1; (c) classified into 5 clusters; and (d) classified into 7 clusters (From Amartur et al., 1992 with permission).

class wins. In this approach, there is no need to determine sensitivities associated with the weighting factors (Lin et al., 1996a; Lin et al. 1996b) that are difficult to determine. Another technique was proposed by Cheng et al., (1996) as Competitive Hopfield Neural Network (CHNN) where the competitive learning rule, Winner-Takes-All (WTA), was utilized to update the weights.

(Chang and Chung, 2001) describe another difficulty of the HNN: the original HNN cannot incorporate contextual information into the segmentation process. They also mention that the robustness of the HNN to noise is low. To overcome this limitation, they introduce Contextual-Constraint-based Hopfield Neural Cube (CCBHNC). The network benefits from a three-dimensional architecture. They incorporated the pixel's feature and its surrounding contextual information into the third dimension.

\subsection{Cellular Neural Network}

Cellular Neural Network (CNN) was proposed by Chua et al. (1988). It is made of a pool of cells that communicate with each other through their neighbours. Consider an $M \times N$ cellular neural network, arranged in $M$ rows and $N$ columns. Each cell connects to the nearest $r \times r$ neighbourhood and the output of each cell is connected with the inputs of the cells in the same neighbourhood. A typical example is presented in Fig. 2. Each cell is 
modelled as an electric circuit. Complete details about the model are found in (Chua et al., 1988a; Chua et al., 1988b; Chua et al., 1988c). The cell equations are as follows (Chua, 1988c). State equation:

$$
C \frac{d v_{x i j}(t)}{d t}=-\frac{1}{R_{x}} v_{x i j}(t)+\sum_{C(k, l) \in N(i, j)} A(i, j ; k, l) v_{y k l}(t)+\sum_{C(k, l) \in N(i, j)} B(i, j ; k, l) v_{u k l}(t)+I
$$

Output equation:

$$
v_{y i j}(t)=\frac{1}{2}\left(\left|v_{x i j}(t)+1\right|-\left|v_{x i j}(t)-1\right|\right)
$$

Input equation:

$$
v_{u i j}(t)=E_{i j}
$$

Constraint conditions:

$$
\left|v_{x i j}(0)\right| \leq 1, \quad\left|v_{u i j}\right| \leq 1
$$

where the subscripts $u, x$, and $y$ denote input, state, and output, respectively. For example, $v_{x i j}$ denotes the state voltage of the cell $(i, j), R_{x}$ is a linear resistor, $I$ is an independent current source and $E_{i j}$ is a time-invariant independent voltage source. Matrixes $A$ and $B$ are considered as feedback template and control template, respectively.

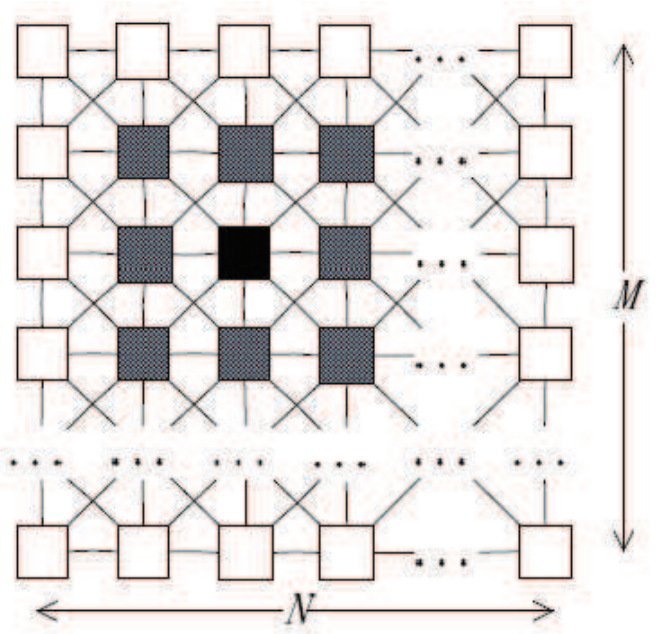

Fig. 2. Cellular Neural Network with a $3 \times 3$ neighbourhood (from Zhang et al., 2007 with permission).

Satisfying the constraints (13) guarantees that the solution always converges to a constant steady state. This means that any transient driven by a given input image vanishes and the network generates a constant dc output. In (Chua et al., 1988b), two medical applications of 
$\mathrm{CNN}$ are described: noise removal and feature extraction (edge detection). CNN has advantages like: 1) parallel calculation on hardware; 2) high calculation speed (almost realtime); and 3) being independent of the image size (Chua et al., 1988b).

CNN is used to develop image segmentation methods (Zhang et al., 2007; Luan et al., 2007; Grassi and Vecchio, 2006). Zhang et al. (2007; 2008) segment urinary images using CNN where the image is segmented after it is enhanced. Selvathi et al. (2010) segment brain tumors in magnetic resonance images using CNN and gradient information.

Yang et al. (1996) and Colodro and Torralba (1996) proposed a fuzzy version of Cellular Neural Networks (FCNN) which is a generalization of CNN to incorporate the uncertainties in human cognitive processes. Chua et al. (1988b) presented applications of FCNN in mathematical morphology and fuzzy inference rule by else action (FIRE) operators like FIRE edge detecting operator. In medical imaging, FCNN was utilized for white blood cell segmentation (Shitong and Min, 2006) and CT liver image segmentation (Wang et al., 2007). In (Shitong and Min, 2006), FCNN was combined with Threshold Segmentation followed by Mathematical Morphology (TSMM) to detect white blood cells. Moreover, Wang et al. (2007) proposed an advanced version of FCNN to segment the CT liver images.

Finally, Gacsádi and Szolgay (2010) proposed a new variational computing based medical segmentation method using CNN. Over the last decade, variational method and partial equations have been widely used for medical image segmentation in algorithms such as level-set (Angelini et al., 2005). This method needs a multi-layered CNN to solve an optimization problem to produce segmentation results.

\subsection{Pulse-coupled Neural Network}

Pulse-Coupled Neural Network (PCNN) is inspired from a cat's visual cortex. A model of the cat's visual cortex was proposed by Eckhorn et al. (1989). Over the past decade, PCNNs have been used for a variety of image processing applications such as segmentation, feature and face extraction, motion detection, and noise reduction (Lindblad and Kinser, 2005). PCNN consists of a pool of neurons where each neuron corresponds to a pixel in the image receiving its local information and the stimuli from its neighbouring neurons. These stimuli are accumulated in the neuron. The neuron fires when the average of stimuli exceeds a dynamic threshold. The dynamic threshold leads to the pulsing nature of PCNN. This network differs from other neural networks in its structure and operation. In particular, the original PCNN does not contain a training phase.

A PCNN neuron shown in Fig. 3 consists of three compartments (Lindblad and Kinser, 2005): the dentritic tree; the modulation; and the pulse generator. There are two types of inputs: Feeding and Linking. Feeding receives an input stimulus from an external source and other neuron outputs while linking only receives them from other neuron outputs. The related formulas are:

$$
\begin{gathered}
F_{i j}[n]=e^{-\alpha_{F} \delta_{n}} F_{i j}[n-1]+S_{i j}+V_{F} \sum_{k l} M_{i j k l} Y_{k l}[n-1] \\
L_{i j}[n]=e^{-\alpha_{L} \delta_{n}} L_{i j}[n-1]+V_{L} \sum_{k l} W_{i j k l} Y_{k l}[n-1]
\end{gathered}
$$

where $L_{i j}$ and $F_{i j}$ are the linking and feeding of the $(i, j)$ neuron corresponding to the $(i, j)$-th pixel in a $2 \mathrm{D}$ image. Both compartments contain a memory of the previous state with 
decays, $e^{-\alpha_{L} \delta_{n}}$ and $e^{-\alpha_{F} \delta_{n}} . V_{L}$ and $V_{F}$ are normalizing constants. $W$ and $M$ matrices include the synaptic weights where each neuron communicates with its neighbouring neurons.

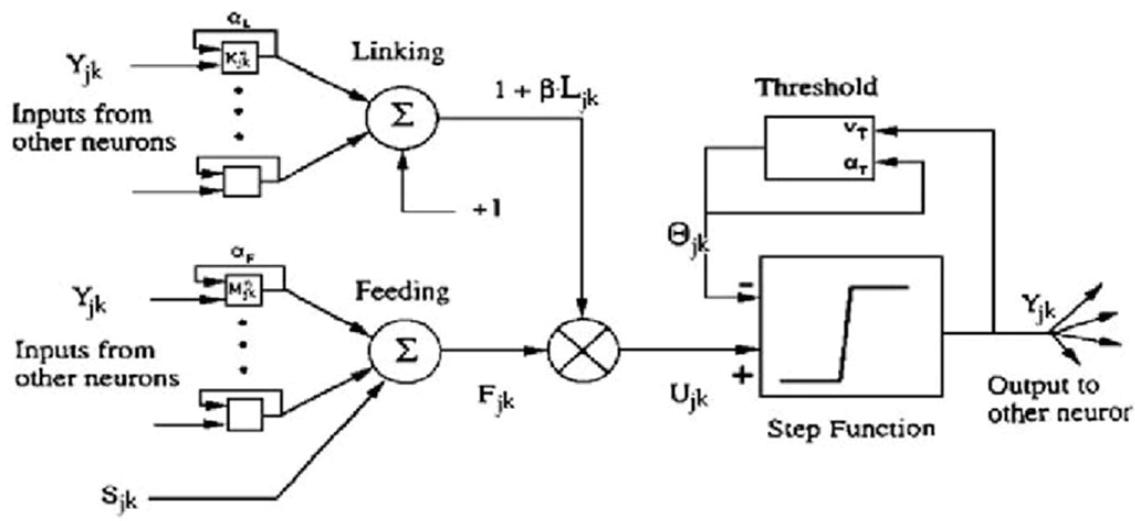

Fig. 3. Schematic of a neuron in PCNN (from Lindblad and Kinser, 2005 with permission).

In the modulation section, the outputs of feeding and linking combine to form a second order equation. The output of modulation section is determined by

$$
U_{i j}[n]=F_{i j}[n]\left\{1+\beta L_{i j}[n]\right\}
$$

where $\beta$ is the linking strength.

In the pulse generator, the output of the previous section is compared to a dynamic threshold, $\theta$, to produce the output of the neuron, $Y$. The dynamic threshold of each iteration is determined by

$$
\Theta_{i j}[n]=e^{-\alpha_{\Theta} \delta_{n}} \Theta_{i j}[n-1]+V_{\Theta} Y_{i j}[n]
$$

where $V_{\Theta}$ is a large constant.

At the beginning, a pixel or a set of neighbouring pixels in the image is considered as a neuron in the PCNN. The values of outputs are all set to zero. A neuron that has an external stimulus will fire in the first iteration. In this situation, the threshold values are high and it will take several iterations in which neurons are allowed to fire again. This iterative process will continue until the user stops it. There is no automatic procedure to stop the PCNN; this is a disadvantage of PCNN. Another disadvantage is a large number of parameters that should be determined appropriately.

The goal of PCNNs for a segmentation process is managing the network such that when a neuron fires, it shows a specific feature like an edge. In addition, similarities between the input pixels may lead to fire the corresponding neurons simultaneously thus showing texture or similar objects.

Keller and McKinnon's (1999) showed that PCNN works well when the segments are almost uniform in intensity. Two kinds of images, magnetic resonance images of the brain and the abdomen and a set of nuclear scintigraphic images of lung, are used for testing the effectiveness of PCNN. They also showed that PCNN did a good job for contrast enhancement. In addition, they found that PCNN was unable to perform a segmentation 
task when the intensity in each segment was not distributed uniformly. They mentioned selection of optimal parameter values as another difficulty of PCNN. For example, it is possible that a set of parameters segment objects properly in a particular image but they do not perform well on a similar image. To this end, Hassanien and Ali (2004) used a Fuzzy Histogram Hyperbolization (FHH) algorithm as a contrast improvement filter for the digital mammogram image segmentation. Then a PCNN performed the segmentation process.

In (Ma et al., 2004), Shannon entropy was used as a criterion to stop the network automatically. In their work, the binary image was used and the information of the original image was not utilized. To overcome to this limitation, Xiao et al. (2009) integrated PCNN with fuzzy mutual information to stop the segmentation process with optimal results. They segmented the CT and ultrasound images with strong robustness against noise. Their method consisted of the following steps:

1. Set initial parameters and iteration number to 1 .

2. Use normalized gray image as an external input to PCNN.

3. Iterate, $\mathrm{n}=\mathrm{n}+1$.

4. Segment the image by PCNN.

5. Compute the value of Fuzzy Mutual Information (FMI). If FMI < FMImax, go to Step 3.

Otherwise, stop the process and report the final result.

In (Shi et al., 2009), a new method was proposed based on improved PCNN and Tsallis entropy. Their method can automatically segment medical images without choosing the PCNN parameters. In the improved PCNN, each neuron is activated only once.

Finally, Fu et al. (2010) proposed an automatic method that integrated the statistical exception maximization (EM) model and PCNN for MRI segmentation. In their method, the EM model does two major works: evaluation of the PCNN image segmentation; and adaptive adjustment of the PCNN parameters for optimal segmentation. Their method has two stages: pre-processing and segmentation. In the former, they retrieve intracranial tissues by a watershed algorithm and in the latter, PCNN segments the image and its parameters are adjusted by the EM model.

\section{Medical image segmentation using feed-forward Neural Networks}

\subsection{Self organized map Neural Network}

Self Organized Map (SOM), introduced by Kohenen (2001), is a feed-forward neural network that has been utilized for medical image segmentation. Sometimes SOM is called Kohenen map. It projects a high dimensional space into a low dimensional space. SOM consists of a 2D array of nodes. Each node is associated with a weight vector, $w_{i}$, of the same dimension and a position in the map space. An unsupervised, iterative learning process finds the weight vector for each node. In each iteration, the most similar node, called the best matching unit (BMU), is found by a similarity measure. A training process considers the neighboring nodes of the BMU and updates their corresponding weight vector as follows (Kohenen, 2001).

$$
w_{i}(t+1)=w_{i}(t)+\alpha(t) h_{c i}(t)\left(x(t)-w_{i}(t)\right)
$$

where $\alpha(t)$ is a monotonically decreasing learning coefficient, $h_{c i}(t)$ is the neighborhood function, typically considered as a Gaussian function. The process repeats for a large number of iterations. SOM preserves the most important topological and metric relationships of the primary data items. 
Although a primary application of SOM is dimension reduction, it has been utilized for medical image segmentation. Rickard et al. (2004) segmented the mammogram images using multi-scale analysis and SOM. Chang and Teng (2007) proposed a two-stage SOM to identify dominant color components and segment a medical image. SOM has been utilized for color reduction of color medical images, although due to the noisy nature of medical images, this method may not be effective. To overcome this problem, Chang and Teng (2007) proposed a two-stage SOM shown in Fig. 4. The first SOM identifies a number of reprehensive colors while the second SOM determines the final dominant colors. A sample result of this method is shown in Fig. 5.

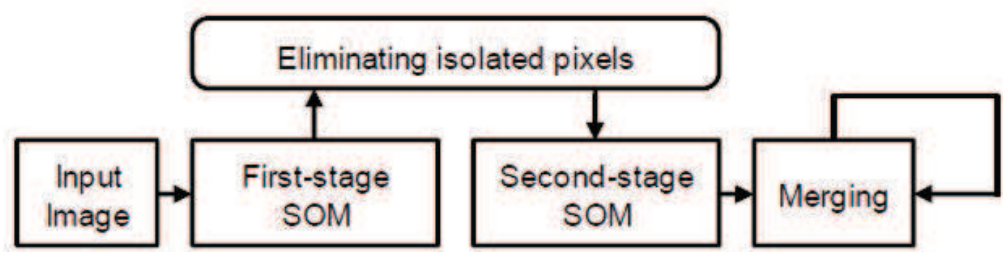

Fig. 4. A flowchart of the two-stage SOM method (from Chang and Teng, 2007 with permission).

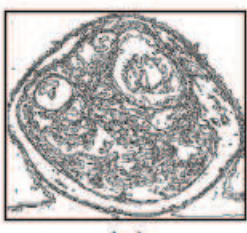

(a)

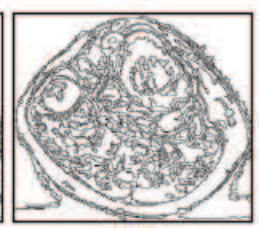

(b)

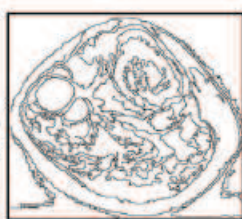

(c)

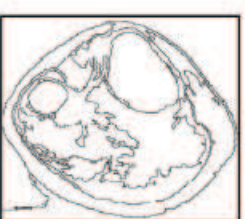

(d)

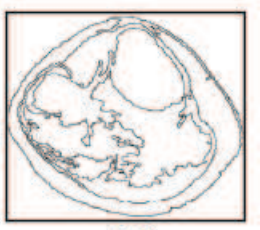

(e)

Fig. 5. Identified segments using merging steps: (a) before merging (b) first merging (c) second merging (d) third merging (e) fourth merging (from Chang and Teng, 2007 with permission).

\subsection{Radial basis function network}

Radial Basis Function (RBF) neural network is another type of feed-forward neural network that uses radial basis functions as activation functions. RBF network consists of three layers: input layer; hidden layer with radial basis function as activation function; and output layer. The output of an RBF network is a linear combination of weighted radial basis functions.

$$
\varphi(\mathbf{x})=\sum_{i=1}^{N} a_{i} \exp \left(-\beta_{i}\left\|\mathbf{x}-\mathbf{c}_{\mathbf{i}}\right\|\right)
$$

where $N$ is the number of neurons in the hidden layer, $\mathbf{c}_{i}$ is the center of neuron $i$, and $a_{i}$ is weight of the linear output neuron. In the training phase, $a_{i}$ and $\beta_{i}$ of each neuron are estimated by a least squares objective function or a regularized objective function. The number of the neuron is set manually.

RBF has been utilized for function approximation and classification (Bishop, 2006). For medical image segmentation, RBF extracts features and classifies image pixels (Sing et al., 
2005). In (Kovacevic, 1997), an RBF network was utilized to segment CT images of the head. In (Halkiotis, 2007), an RBF network was used to detect clustered microclasifcations in digital mammograms automatically.

\subsection{Multi-layer perceptron Neural Network}

Multi-layer Perceptron Artificial Neural Network (MLP-ANN) is used in a wide range of applications including function approximation, feature extraction, optimization, compression, and classification (Bishop, 2006). It is appropriate for medical imaging applications because of the following reasons: 1) For an MLP-ANN with a hidden layer, the output is a nonlinear function of a linear combination of the neuron outputs in the hidden layer which themselves are nonlinear functions of linear combinations of the input variables. This allows the network to construct parametric nonlinear functions of the input features. The parameters of the network are estimated by optimizing an objective function (usually the mean square error between the desired and actual outputs) by a back-propagation algorithm. 2) Due to the correlation between the input features, the intrinsic dimensionality of the input feature space is usually smaller than the number of input features. When the number of neurons in the hidden layer is smaller than that of the input layer, it means that the hidden layer represents a lower dimensional feature space, which can have the same number of dimensions as that of the intrinsic feature space. 3) Depending on the desired output, some of the features may be irrelevant. An MLP-ANN automatically ignores them by assigning zero (or very small weights) to them. In a sense, it does the job of feature selection.

Requirements and limitations of MLP-ANN are the following. First, a proper configuration of the network with best generalization should be established by choosing proper numbers of hidden layers and neurons. Secondly, the objective function of the network is not always convex. Therefore, the gradient descend algorithm may find a local minimum instead of the global minimum. To overcome the first problem and acheive best generalization, available data is divided into training, testing, and validation datasets, where the error is controlled for the validation data.

Some image segmentation algorithms have benefited from MLP-ANN (Jabarouti Moghaddam et al., 2009; Jabarouti Moghaddam and Soltanian-Zadeh, 2009; Magnotta et al., 1999; Powell et al., 2008). The method developed by Magnotta et al. (1999) uses Image Intensity Values (IIVs) of the neighboring voxels as the image features. They classify the voxels into two classes - being inside and outside of a desired structure. They design an MLP-ANN for each structure. Since their image features do not contain a shape representation, they need to use a large training set. The IIVs solve the segmentation problem for the high-resolution MRI they utilized. Recently, Powell et al. (2008) have further developed their previous algorithm (Magnotta et al., 1999) using 9 IIVs along with the largest gradient, a probabilistic map, and the IIVs along each of the three orthogonal axes. They use high-resolution images the same as (Magnotta et al., 1999) for the segmentation of the brain structures (putamen, caudate, thalamus, and cerebellar regions of interest). They use 15 image volumes, collected using two protocols, T1-weighted and T2-weighted, in the training set. Their approach does not include any shape representations. For dimension reduction of the IIV features, the IIVs along the largest gradient are utilized. In low resolution MRI with intensity inhomogeneity inside the structures, these features may not work well. 
Jabarouti Moghaddam et al. (2009) used Geometric Moment Invariants (GMIs) (Lo and Don, 1989) to improve the differentiation between the brain regions compared to the IIVs. In (Mangin et al., 2004), shape descriptors were defined based on the moments of the voxel coordinates for morphometry of the cortical sulci. GMIs characterize the underlying anatomical structures calculated for each voxel. They are eleven moment invariants calculated from the first-order, second-order, and third-order 3D-regular moments. They are invariant to translation and rotation. They designed an MLP-ANN to segment putamen. The image features were the GMIs for white matter (WM) and gray matter (GM) tissues along with the neighboring IIVs. The output of the network was a scalar that took two values $(-1$, 1) for indicating inside of the structure and its outside. The GMIs were calculated only for one scale. However, considering only one scale may not be sufficient to distinguish all parts of the putamen. In addition, the method depends on the tissue segmentation results as it calculates the GMIs of the WM and the GM maps.

Jabarouti Moghaddam and Soltanian-Zadeh (2009) developed a two-stage method for the segmentation of the brain structures as shown in Fig. 6. In the first stage, GMIs were used along voxel intensity values as an input feature and signed distance function of a desired structure as an output of the network. In this stage, there is a network for each scale of GMIs. Fig. 7 shows the MLP-ANN used for the first stage.
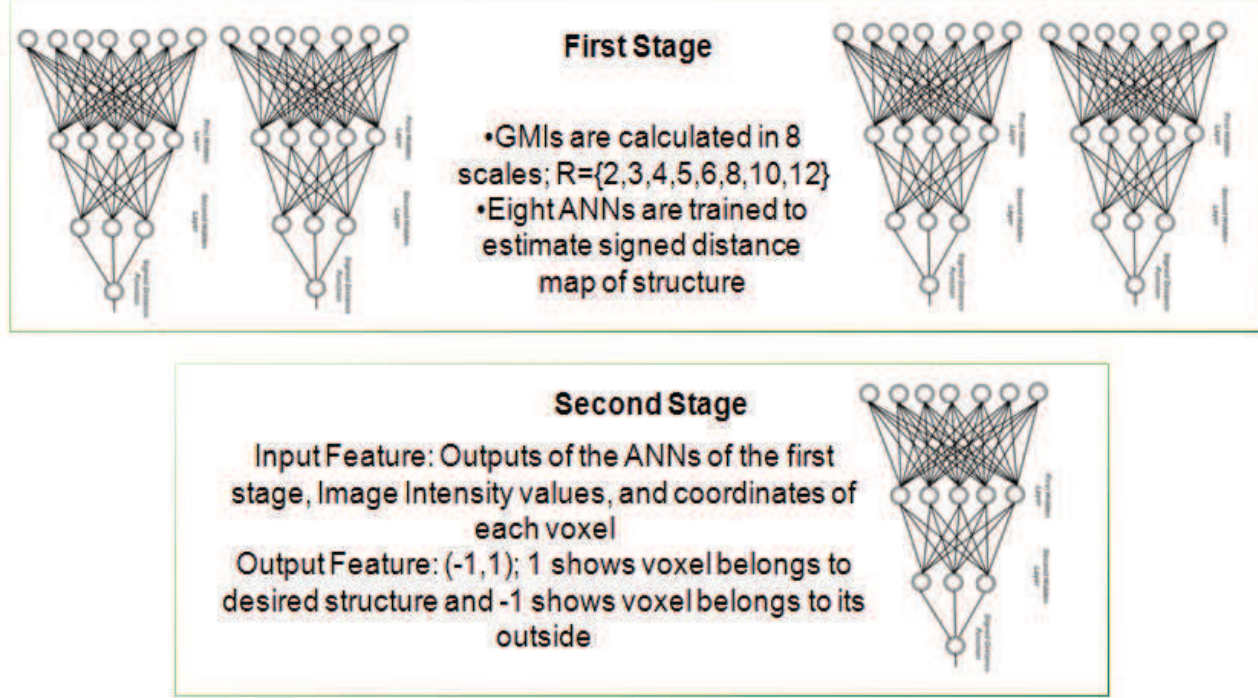

Fig. 6. A flowchart of the two-stage method.

In the first stage, each input feature vector includes the neighboring voxel intensity values, GMIs, and coordinates of voxels. More formally, at voxel $x$, the input feature vector $a(x)$ is composed of three vectors $a(x)=\left[a_{1}(x) a_{2}(x) a_{3}(x)\right]$ where $a_{1}(x)$ is for voxel intensity values, $a_{2}(x)$ is for coordinate of voxel $x$, and $a_{3}(x)$ is for GMIs. 


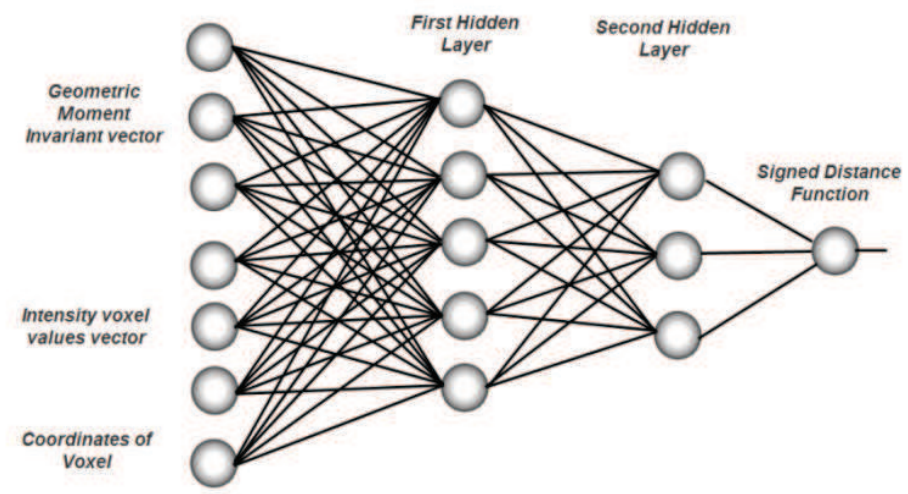

Fig. 7. MLP-ANN architecture of the first stage. Input feature contains three components GMIs, voxel intensity values, and coordinates of voxels. There are two hidden layers. There are 33 neurons in the input layer, 17 neurons in the first hidden layer, 7 neurons in the second hidden layer, and a single neuron in the output. The output is designed to approximate the singed distance function of the structure.

The vector $a_{1}(x)$ contains the neighboring voxel intensity values of voxel $x$. We define $a_{1}(x)$ by considering the neighbouring voxels along the orthogonal axes ( \pm 3 voxels in each direction) of the 3D-space. Therefore, the dimension of $a_{1}(x)$ is 13 (Fig. 8).

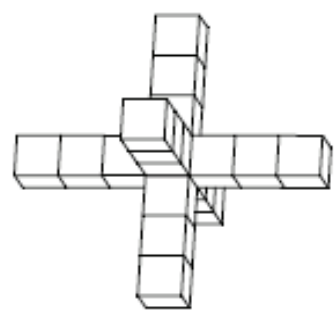

Fig. 8. Neighboring voxel intensity values that are considered for each voxel of the training data sets as a part of the input feature vector.

The vector $a_{2}(x)$ consists of the coordinates of voxels in the Cartesian coordinate system. This can help the network to find relations between the voxels of a specific structure. The brain structures may have discontinuous image intensities inside the structure. Therefore, considering coordinates of the voxels in the input feature vector can help with the training of the network.

The vector $a_{3}(x)$ contains the GMIs at voxel $x$. These features include rich geometric properties, represent the underlying anatomical structures, and can distinguish voxels that are inside the structure from those that are outside of the structure. Lo and Don (1989) explicitly define 11 moment invariants consisting of the first, second, and third order 3D-regular moments. The input feature vector is made of 33 features: 19 features for voxel intensity values; 11 features for GMIs; and 3 features for voxel coordinates in the training data sets. 
For shape representation, a signed distance function of the structure of interest is used as the output. The signed distance function is a subset of implicit functions in shape representation. It is defined to be positive in the exterior, negative in the interior, and zero on the boundary. Near the boundaries, the value of this function decreases and far from the boundaries, the value of this function increases.

The network is trained using the backpropagation algorithm and voxels inside the structure of interest. Mean Square Error (MSE) is calculated and compared until it reaches an asymptotic value. The range of MSE in the first stage is from 0.02 to 0.03 . Each training network takes 1 day so we need almost 9 days for training the networks used in both stages. Although this is a relatively long time but it is only performed once.

Outputs of the first stage can distinguish different parts of the structure. The 8 outputs of the first stage must be combined to generate the final result. As such, another MLP-ANN was used to combine the 8 outputs of the networks in the first stage. In the second stage, we use the network as a classifier, not as a function approximator. Our task in this stage is to classify voxels into two classes - being inside or outside of the structure. The results of the networks in the first stage are smoothed using a mean filter with a kernel size of 3 . This eliminates the outlier voxels. The input features consist of the 8 smoothed versions of the outputs of the first stage, 19 image intensity values of the neighboring voxels, and 3 voxel coordinates in the Cartesian coordinate system. They generate a 30-dimensional feature vector. The network setting used in this stage is the same as that used in the first stage. The lower the error in this stage, the better the results. The resulting network activation function is thresholded at 0 to define the structure of interest. The voxels with outputs larger than 0 define the structure of interest and the voxels with outputs smaller than 0 define the outside region.

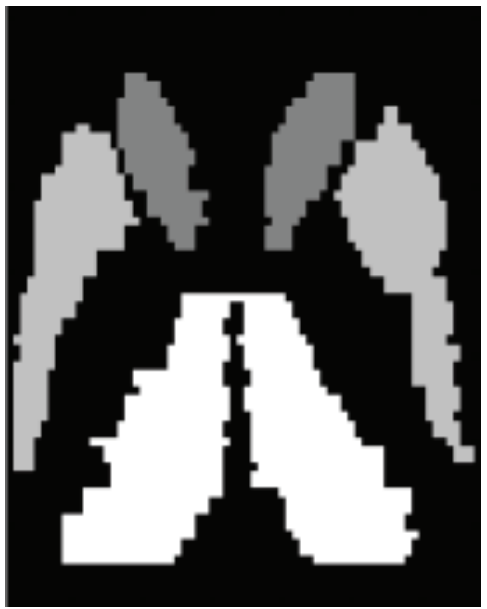

(a)

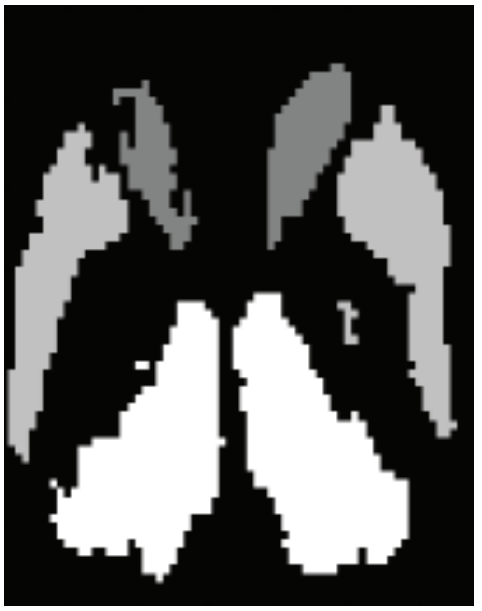

(b)

Fig. 9. (a) Manual segmentation by experts. (b) Outputs of the second stage showing the final segmentation result for a sample slice. 
The results of the second stage and a 3D visualization of the putamen, caudate, and thalamus are shown in Figures 9 and 10, respectively.

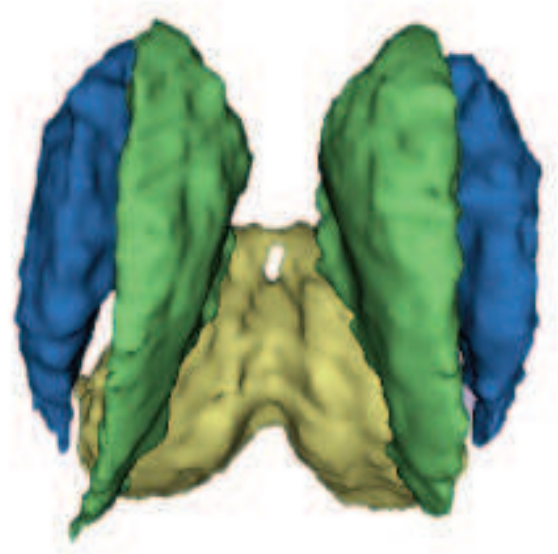

(a)

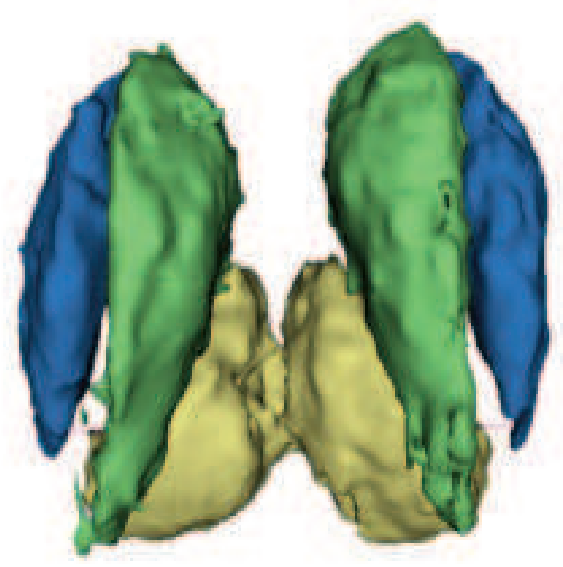

(b)

Fig. 10. 3D visualization of the outputs of three networks used for three structures. Caudate is shown in green, putamen is shown in blue, and thalamus is shown in yellow.

\section{Conclusion}

In this chapter, we presented several methods for medical image segmentation based on artificial neural networks. The networks were categorized into feedback and feed-forward networks. Among the feedback networks, Hopfield, Cellular, and Pulse-Coupled networks have been used. Among the feed-forward neural networks, Self Organizing Map (Kohenen), Multi-Layer Perceptron, and Radial Basis Function neural networks have been utilized.

In Section 2, the Hopfield network was described as a map between the image pixels and their labels. The network is easily implemented in hardware and used in real-time applications. On the other hand, the network is fully connected and it is not applicable for $3 \mathrm{D}$ or huge 2D medical image segmentation due to its large number of parameters. In addition, for object recognition, it is not essential that each pixel connects to all other pixels. Indeed, it may be ineffective to incorporate information of all other pixels to label a pixel. Next, Cellular Neural Network was discussed. In this network, the information of the neighboring pixels is included in pixel segmentation process which is an advantage relative to the Hopfield network. Due to this advantage, it is applicable for 3D or huge 2D medical image segmentation. At the end of the section, the Pulse Coupled Neural Network was explained. This network differs from other neural networks in its structure and operation. In particular, the original PCNN does not contain a training phase. The lack of a stopping method is a challenge for PCNN.

In Section 3, the SOM neural network was explained as a feed-forward network. It is usually utilized for dimension reduction. As discussed, it has been used for color reduction of 
medical images. The next network discussed was the RBF neural network. This type of neural network is usually useful for function approximation and classification. Finally, applications of the Multi-Layer Perceptron neural network were discussed. This type of neural network is effective for segmenting deep brain structures in 3D. Its high speed makes it appropriate for real-time applications. Due to its speed and accuracy, it has a bright future for medical image segmentation applications.

\section{References}

Amartur, S. C.; Piraino, D. \& Takefuji, Y. (1992). Optimization neural networks for the segmentation of magnetic resonance images. IEEE Transactions on Medical Imaging, 11(2): 215-220.

Angelini, E. D.; Jin, Y. \& Laine, A. (2005). State-of-the-art of levelset methods in segmentation and registration of medical imaging modalities. The Handbook of Medical Image Analysis 3.

Bishop, C. M. (2006). Pattern Recognition and Machine Learning, Springer.

Chang, C. Y. \& Chung, P. C. (2001). Medical image segmentation using a contextualconstraint-based Hopfield neural cube. Image and Vision Computing, 19(9-10): 669678.

Chang, P. L. and W. G. Teng (2007). Exploiting the self-organizing map for medical image segmentation, 20 th IEEE international Symposium on Computer-Based Medical Systems (CBMS'07), Maribor, 281:288.

Cheng, K.-S.; Lin, J.-S. \& Mao, C.-W. (1996). Application of competitive Hopfield neural network to medical image segmentation. IEEE Transactions on Medical Imaging 15(4): 560-561.

Chua, L. O. \& Yang, L. (1988a). Cellular neural networks, IEEE international Symposium, Circuits and Systems.

Chua, L. O. \& Yang, L. (1988b). Cellular neural networks: Applications. IEEE transactions on circuits and systems, 35(10): 1273-1290.

Chua, L. O. \& Yang, L. (1988c). Cellular neural networks: Theory. IEEE transactions on circuits and systems, 35(10): 1257-1272.

Colodro, F. \& Torralba, A. (1996). Cellular neuro-fuzzy networks (CNFNs), a new class of cellular networks, Proceeding of $5^{\text {th }}$ IEEE on Fuzzy Systems, New Orleans, LA, USA.

Eckhorn, R.; Reitboeck, H. J., Arndt, M. \& Dicke, P. (1989). Feature linking via stimulusevoked oscillations: Experimental results from cat visual cortex and functional implications from a network model, International Joint Conference on Neural Network, Washington, DC, USA.

Fu, J. C.; Chen, C. C., Wong S.T.C. \& Li, I.C. (2010). Image segmentation by EM-based adaptive pulse coupled neural networks in brain magnetic resonance imaging. Computerized Medical Imaging and Graphics 34(4): 308-320.

Gacsádi, A. \& Szolgay, P. (2010). Variational computing based segmentation methods for medical imaging by using CNN, $12^{\text {th }}$ international Workshop on Cellular Neural Networks and Their Applicaiton (CNNA), 1:6. 
Giménez-Martínez, V. (2000). A modified Hopfield auto-associative memory with improved capacity. IEEE Transactions on Neural Networks, 11(4): 867-878.

Grassi, G. and P. Vecchio (2006). Cellular neural networks for object-oriented segmentation. Research in Microelectronics and Electronics 2006, Otranto.

Halkiotis, S.; Botsis, T. \& Rangoussi, M. (2007). "Automatic detection of clustered microcalcifications in digital mammograms using mathematical morphology and neural networks." Signal Processing, 87(7): 1559-1568.

Hassanien, A. E. \& Ali J.M. (2004). Digital mammogram segmentation algorithm using pulse coupled neural networks, Third International Conference on Image and Graphics (ICIG'04), Hong Kong.

Hopfield, J. J. (1982). Neural networks and physical systems with emergent collective computational abilities. Proceedings of the National Academy of Sciences of the United States of America, 79(8): 2554-2558.

Jabarouti Moghaddam, M.; Rahmani, R. \& Soltanian-Zadeh, H. (2009). Automatic segmentation of putamen using geometric moment invariants. The 15th Iranian Conference on Biomedical Engineering, Mashad, Iran.

Jabarouti Moghaddam, M. \& Soltanian-Zadeh, H. (2009). Automatic segmentation of brain structures using geometric moment invariants and artificial neural networks. Information processing in medical imaging : 21st International Conference on Information Processing in Medical Imaging (IPMI'09), Williamsburg, VA, 21: 326-337.

Keller, P. E. \& McKinnon, A.D. (1999). Segmentation of medical imagery with pulse-coupled neural networks, International Joint Conference on Neural Network, Washington, DC, USA, IEEE.

Kohenen, T. (2001). Self-Organizing Maps, Springer.

Kovacevic, D. \& Loncaric, S. (1997). Radial basis function-based image segmentation using a receptive field, Processing of $10^{\text {th }}$ IEEE Symposium on Computer-Based Medical Systems, Maribor, Slovenia.

Lin, J.-S.; Cheng, K.-S. \& Mao, C.-W. (1996a). Modified Hopfield neural network with fuzzy c-means technique for multispectral MR image segmentation, Processings of International IEEE Conf., Lausanne, Switz, 1,327:330.

Lin, J.-S.; Cheng, K.-S. \& Mao, C.-W. (1996b). a fuzzy hopfield neural network for medical image segmentation, IEEE Transactions on Nuclear Science 43(4 PART 2): 2389-2398.

Lin, J.-S.; Cheng, K.-S. \& Mao, C.-W. (1996c). "Multispectral magnetic resonance images segmentation using fuzzy Hopfield neural network." International Journal of BioMedical Computing 42(3): 205-214.

Lindblad, T. \& Kinser, J.M. (2005). Image Processing Using Pulse-Coupled Neural Networks, Springer.

Lo, C.-H. \& Don, H.-S. (1989). 3-D moment forms: Their construction and application to object identification and positioning. IEEE Transactions on Pattern Analysis and Machine Intelligence, 11(10): 1053-1064. 
Luan, Q.X.; Li, X., Kang, J.Y. \& Zhi, Z. (2007). Analysis of dental plaque by using cellular neural network-based image segmentation. Chinese journal of stomatology 42(12): 720-722.

Ma, Y. D.; Liu, Q. \& Zhi-Bai, Q. (2004). Automated image segmentation using improved PCNN model based on cross-entropy, Proceeding of 2004 International Symposium on Intelligent Multimedia, Video and Speech Processing, Hong Kong, China.

Magnotta, V. A.; D. Heckel, Andreasen, N.C., Corson, P.W., Ehrhardt, J.C. \& Yuh, W.T.C. (1999). Measurement of brain structures with artificial neural networks: Two- and three-dimensional applications. Radiology 211(3): 781-790.

Mangin, J. F.; F. Poupon, Duchesnay, E., Riviere, D., Cachia, A., Collins, D.L., Evans, A.C., Regis, J. (2004). Brain morphometry using 3D moment invariants. Medical Image Analysis, 8(3): 187-196.

Powell, S.; V. A. Magnotta, Johnson, H., Jammalamadaka, V.K., Pierson, R. \& Andreasen, N.C. (2008). Registration and machine learning-based automated segmentation of subcortical and cerebellar brain structures. NeuroImage 39(1): 238-247.

Rickard, H. E.; Tourassi, G. D. \& Elmaghraby, A.S. (2004). Breast segmentation in screening mammograms using multiscale analysis and self-organizing maps, Proceedings of the 26th Annual International Conference of the IEEE EMBS, San Francisco, CA.

Selvathi, D.; Selvaraj, H. \& Selvi, S.T. (2010). Hybrid approach for brain tumor segmentation in magnetic resonance images using cellular neural networks and optimization techniques. International Journal of Computational Intelligence and Applications 9(1): 17-31.

Shi, W.; Miao, Y., Zhanfang, C. \& Hongbiao, Z. (2009). Research of automatic medical image segmentation algorithm based on Tsallis entropy and improved PCNN, Internatioal Conference on Mechatronics and Automation, Changchun.

Shitong, W. \& Min, W. (2006). A new detection algorithm (NDA) based on fuzzy cellular neural networks for white blood cell detection. IEEE Transactions on Information Technology in Biomedicine, 10(1): 5-10.

Sing, J. K., D. K. Basu, Nasipuri, M. \& Kundu, M. (2005). Self-adaptive RBF neural networkbased segmentation of medical images of the brain, Proceedings of International Conference on Intelligent Sensing and Information Processing.

Wang, S.; Fu, D., Xu, M. \& Dewen, H.U. (2007). Advanced fuzzy cellular neural network: Application to CT liver images." Artificial Intelligence in Medicine 39(1): 65-77.

Wu, Y. \& Pados, D.A. (2000). A feedforward bidirectional associative memory. IEEE Transactions on Neural Networks, 11(4): 859-866.

Xiao, Z.; Shi, J. \& Chang, Q. (2009). Automatic image segmentation algorithm based on PCNN and fuzzy mutual information, IEEE internatioal Conference on Computer and Information Technology, 241:245.

Yang, T.; Yang, L.-B., Chai, W.W. \& Chua, L.O. (1996). Fuzzy cellular neural networks: theory, $4^{\text {th }}$ IEEE International Workshop on Cellular Neural Network, Seville, Spain. 
Zhang, Z.; Xia, S.R. \& Duan, H.L. (2007). Cellular neural network based urinary image segmentation. 3th International Conference on Natural Computation (ICNC), Haikou, Hainan.

Zhang, Z. C.; Xia, S.R. \& Duan, H.L. (2008). Cellular neural network based urinary sediment image segmentation. Zhejiang Daxue Xuebao (Gongxue Ban)/Journal of Zhejiang University (Engineering Science) 42(12): 2139-2144. 


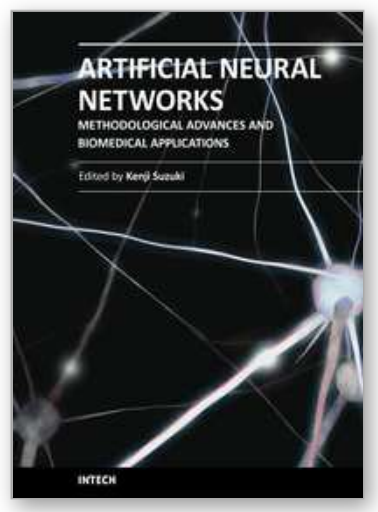

\section{Artificial Neural Networks - Methodological Advances and Biomedical Applications}

Edited by Prof. Kenji Suzuki

ISBN 978-953-307-243-2

Hard cover, 362 pages

Publisher InTech

Published online 11, April, 2011

Published in print edition April, 2011

Artificial neural networks may probably be the single most successful technology in the last two decades which has been widely used in a large variety of applications in various areas. The purpose of this book is to provide recent advances of artificial neural networks in biomedical applications. The book begins with fundamentals of artificial neural networks, which cover an introduction, design, and optimization. Advanced architectures for biomedical applications, which offer improved performance and desirable properties, follow. Parts continue with biological applications such as gene, plant biology, and stem cell, medical applications such as skin diseases, sclerosis, anesthesia, and physiotherapy, and clinical and other applications such as clinical outcome, telecare, and pre-med student failure prediction. Thus, this book will be a fundamental source of recent advances and applications of artificial neural networks in biomedical areas. The target audience includes professors and students in engineering and medical schools, researchers and engineers in biomedical industries, medical doctors, and healthcare professionals.

\section{How to reference}

In order to correctly reference this scholarly work, feel free to copy and paste the following:

Mostafa Jabarouti Moghaddam and Hamid Soltanian-Zadeh (2011). Medical Image Segmentation Using Artificial Neural Networks, Artificial Neural Networks - Methodological Advances and Biomedical Applications, Prof. Kenji Suzuki (Ed.), ISBN: 978-953-307-243-2, InTech, Available from:

http://www.intechopen.com/books/artificial-neural-networks-methodological-advances-and-biomedicalapplications/medical-image-segmentation-using-artificial-neural-networks

\section{INTECH}

open science | open minds

\section{InTech Europe}

University Campus STeP Ri

Slavka Krautzeka 83/A

51000 Rijeka, Croatia

Phone: +385 (51) 770447

Fax: +385 (51) 686166

www.intechopen.com

\section{InTech China}

Unit 405, Office Block, Hotel Equatorial Shanghai

No.65, Yan An Road (West), Shanghai, 200040, China 中国上海市延安西路65号上海国际贵都大饭店办公楼405单元

Phone: +86-21-62489820

Fax: +86-21-62489821 
(C) 2011 The Author(s). Licensee IntechOpen. This chapter is distributed under the terms of the Creative Commons Attribution-NonCommercialShareAlike-3.0 License, which permits use, distribution and reproduction for non-commercial purposes, provided the original is properly cited and derivative works building on this content are distributed under the same license. 